
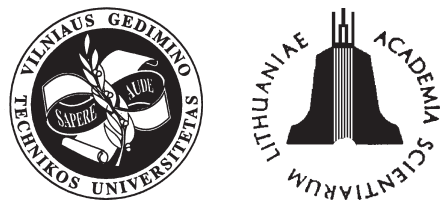

ISSN 1648-4142 TRANSPORT

http:/www.vtu.lt/english/editions

TRANSPORT - 2005, Vol XX, No 3, 99-105

\title{
VEHICLE SPEED CONTROL USING ROAD BUMPS: PART 2
}

\author{
${ }^{1}$ Sunday Ayoola Oke, ${ }^{2}$ Tajudeen Abiola Ogunniyi Salau, ${ }^{3}$ Adebayo Oludele Adeyefa \\ ${ }^{1}$ Dept of Mechanical Engineering, University of Lagos, Akoka-Yaba, Lagos, Nigeria. \\ Corresponding author. E-mail: sa_oke@yahoo.com \\ ${ }^{2,3}$ Dept of Mechanical Engineering, University of Ibadan, Ibadan, Nigeria
}

Received 2004-12-20; accepted 2005-03-25

\begin{abstract}
In a previous publication in this journal the framework for the development of a model for the road bump problem was laid. The current paper focuses on the aspect of determining the effective distance between two consecutive road bumps developing equations governing the velocity and the wavelength of the motion of the vehicle. The wave equation is re-modelled connecting the isolation factor, linear velocity, wavelength and the frequency of motion of the vehicle over a road bump. A new set of equations is developed to extend the framework earlier presented in Salau et al. The feasibility of the model is demonstrated by its behaviour in graph and simulated data generation.
\end{abstract}

Keywords: vehicle control, road bumps speed limit, accidents prevention, effective distance.

\section{Introduction and literature review}

Road bumps research has recently emerged as a new star in transportation studies. A new perspective taken by some researchers has been the design of road bumps in such a way to control excessive speeding with the use of some scientific indicators. Primarily the use of isolation frequency factor and its relationship with the frequency equation towards determining the effective distance between two road bumps is well established in the literature.

There have been several attempts by scholars to investigate into the different aspects of road research. Extensive studies that have been carried out are not limited to pedestrian safety, but include the effect of some external faces on the vehicles that move on the road. In this section we will review related studies on road research to the current subject and then streamline the review effort to the research carried out till the date of vehicle speed control with emphasis on recent work by Salau et al. [8].

Rubinstein and Hition [9] carried out an interesting research with a focus on developing a detailed multi-body model for dynamic simulation of off-road tracked vehicles. In particular a three dimensional multi-body simulation model for simulating the dynamic behaviour of track off-road vehicles was developed using LMS - DADS simulation program. The model was first applied to M113 armored carrier. Simulation results under various road conditions were compared with the results of a super-element-base- model. It was concluded that the influence of the track dynamics and the soil-link interaction on the vehicle dynamics can be better predicted with the newly developed model. While the above work relates the various components of the vehicle such as Chasis, the wheel arms and the wheel, no efforts were made to relate all these components to the road bump. The effects of the road bump on the component were not discussed.

In a study by Berry et al [1] the influence of speed, grade and mass during simulated off road bicycling was investigated. The purpose of the investigation was to examine the effects of bicycle mass, speed and grade on oxygen consumption, heat rate (HR) and ratings of perceived exertion (RPE) during a simulated offroad riding paradigm. Nine adult subjects with mean \pm SD age mass and max of $26,1 \pm 5,6$ years, $71,7 \pm$

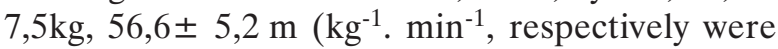
trained to ride a fully suspended Trek Y-22 mountain bike on a treadmill with a $3,8 \mathrm{~cm}$ bump affixed to the belt. Riders completed a maximum of nine separate trials encompassing three different bike masses $(11,6$, 12,6 and $13,6 \mathrm{~kg}$ ).

Yet in another study Lee et al [6] assessed the driving performance of older adult drivers by comparing on-road with simulated driving. To validate a laboratory-based $\mathrm{n}$ simulator on measuring on-road driving performance 129 older adult drivers were assessed with both the simulator and an on road test. The driving performance of the participants was 
gauged by appropriate and reliable age-specific assessment criteria which were found to be negatively correlated with age. Using principal component analysis there were two performances in simulated driving and the no-road assessment. The results supported the validity of the driving simulator and it is a safer and more economical method than the on-road testing to assess the driving performance of older adult drivers. This work investigates into speed control but did not mention the relationship of road bumps to the framework hence in justification of the present work.

The optimization of road vehicle passive suspension systems was studied by Naude and Suyman, [7]. The model developed is linked with a mathematical optimization algorithm in order to enable the optimization of vehicle-designed parameters with the minimization of a well defined objective function. In part 1 of the paper the concept of multi-disciplinary design optimization is discussed. This is followed by the presentation of up to six degrees of freedom vehicle model developed for the study and a discussion of the specific gradient-based optimization algorithm selected for the optimization.

In particular the derivation of the set of the second order differential equations describing the acceleration of the different solid bodies of the vehicle model is presented. In order to perform the optimization of the non-linear suspension component characteristics a six piece-wise continuous and linear approximation is used which is also described in this paper. Part 2 of the study will outline the simulation programme and the qualification of the programme. It will also present a typical case study where the proposed optimization methodology is applied to improve the damper characteristics of a specific vehicle. The study is limited to a suspension system and does not relate to a road bump or what effect it would have on the suspension system.

In a study on how to improve the vehicle performance with active suspension using a road-sensing algorithm Kim et al [4] presents the development of the road sensing system and its application to active suspension system using preview road information. The road-sensing system which can robustly reconstruct the road input profiles from the intermixed data with the vehicle's dynamic motion, is implemented using the composite-sensor system with the optimally shaped transfer function. The study does not relate to the road bump which is the subject of the current work.

Das [2] conducted a field study to observe the standing orientation and behaviour among a group of meat type goats during transit by road. The goats were not observed to orient themselves opposite to the truck direction of travel. Although the animals changed their orientation frequently, apparently to maintain balance, there was a clear bias against the perpendicular and diagonal orientations. Mean frequencies for the various behaviours were bleating $(1,3)$, jumping $(0,7)$, pushing $(0,9)$, fighting $(0,7)$, falling $(3,1)$, urination $(0,5)$, defecation $(0,3)$ and rumination $(0,1)$. Jumping and bleating mostly occurred at the start of the journey. Virtually, all fallings were associated with the driver's changing speed of a vehicle by braking $(43,4 \%)$, concerning $(32,4 \%)$, speed bump $(12,3 \%)$ and acceleration $(11,9 \%)$. The study is also limited to accident prevention on roads, but lacks any linkage to road bumps.

In the study on the driving anger expression inventory a measure of how people express their anger on the road. Dieffenbachia et al. [3] identified four ways people express their anger when driving. Verbal aggressive expression $(=0,88)$ assesses verbally aggressive expression of anger (e.g. yelling or cursing at another driver), personal physical aggressive expression $(0,81)$, the ways the person uses him/herself to express anger (e. g. trying to get out and tell off or have a physical fight with another driver); use of the vehicle to express anger $(=0,86)$, the ways the person uses his/her vehicle to express anger (e. g., flashing lights or cutting another driver off in anger); and adaptive/constructive expression $(=0,90)$, the ways the person copes positively with anger (e. g., focuses on safe driving or tries to relax). Aggressive forms can be summed into total aggressive expression index $(=0,90)$. Aggressive forms of expression correlated positively with each other $(r \mathrm{~s}=0,39-0,48)$, but were uncorrelated or correlated negatively with adaptive/ constructive expression ( $r \mathrm{~s}=-0,02$ to $-0,22)$.

In a related study Salau et al. [8] presented a set of equations that would be useful in determining the effective distance between two road bumps in the control of vehicle speeds. While this study is original research in this direction, the use of a simplified velocity formula relating frequency to wavelength is significant limitation in the study. This limitation arises as a result of the model inability to cope with complex situations demanding the application of more than one function in its computation. As such an imprecise model that is limited in its application results, in other to correct this shortcoming we focus on the velocity equation and expand the framework to incorporate some functional dimensions.

The development of models and empirical solutions of solving the road bump problem has recently gained the attention of researchers on transportation and highway engineering. One of the late developments is the modelling of the road bump problem in a way that would reveal certain important road bump 
parameters. The isolation factor clearly stands as an important parameter used for this purpose. Arising from this there is the application of the wave equation in calculating the effective distance between two road bumps. Unfortunately, in many research explorations the focus has been the use of the simplified wave equation. While this has served various purposes, it has several limitations in terms of its inappropriateness for solving complex problems. In this work detailed investigation into the wave equation is pursued in such a way that researchers would be able to solve complex problems. However, some new developments leading to the velocity equation are also presented. In particular, the wave equation is connected with the velocity, displacement frequency and wavelength time.

\section{The model framework}

The diagram below shows the representation of a road with a dented-rectangular road bump for enforcing vehicle speed limits.

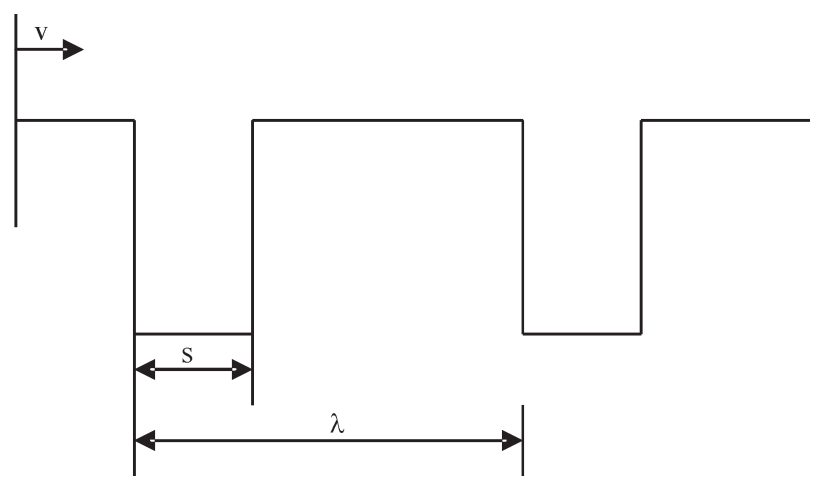

Fig 1. Hollow rectangular shaped bump

In the determination of effective distance covered by the vehicle that passes over the road bump we know that the application of the wave equation in engineering studies finds its way into the problem since the vehicle is assumed to approach the road bump at velocity (v), with frequency (f) generated from the motion, and wavelength of $(\lambda)$ as a result of the motion. Thus, the simple velocity-wavelength equation labelled (1) below applies.

$$
v=f \lambda .
$$

This could be re-written with wavelength made the subject of the expression. Thus, we have:

$$
\lambda=\frac{v}{f},
$$

where the precise definition of terms are: $\lambda$-distance between successive points/wavelength of the motion; $f$ - frequency at which vehicle is moving over road bumps; $v$ - velocity at which the vehicle travels over the road bump.

Note that $f$ can be determined from safe frequency got from the graph of an isolation factor against frequency. Now, referring to Fig 1, we could determine the effective distance moved by the vehicle over the road bump by subtracting the value of "s" from that of " $\lambda$ ". This is expressed as:

$$
\text { Effective distance }=\lambda-s=\frac{v}{f}-s .
$$

The above equation for the effective distance between two road bumps contains an element of velocity that could be modelled mathematically into a more reliable form than its current state is. Thus, the work by Salau et al. [8] ends here while the extension made to the work starts here. In other words the value of the velocity (v) obtained at this stage was used in the work by Salau et al. [8] for further computation, while we developed a new formula for the velocity for use in the model by Salau et al. [8].

Before we could develop a new formula for the velocity we need to assume that the vehicle passes through a medium of a particular refractive index. In this case, air. Thus, let us consider the possible movement of a wave in the media. Let the refractive index for each medium be defined by the distance

$$
\phi(x)= \begin{cases}0 & x<0, \\ 1 & 0<x \leq b, \\ 4 / 3 & b<x \leq a, \\ 3 / 2 & a<x \leq c, \\ 0 & x>c .\end{cases}
$$

But the velocity-wavelength relationship is usually expressed as $V=f \lambda$.

We can introduce parameters $\phi_{(\mathrm{x})} T(t)$ into the equation since we are considering the movement of the wave in a medium. Thus, we can say

$$
V=f_{0} \lambda_{0} \phi_{(x)} T(t)
$$

where $f_{0}$ and $\lambda_{0}$ are the frequency and wavelength of the wave in vacuum. But note $f_{0}$ is constant for particular wavelength. Rearranging equation (5), we have:

$$
\frac{V}{f_{0} \lambda_{0}}=\varphi_{(x)}(T(t)) \text {. }
$$

For the simplicity of modelling and as a matter of research strategy 


$$
\text { let } \frac{V}{f_{0} \lambda_{0}}=\varphi,
$$

therefore $\varphi=\phi(x) T(t)$.

Now we can apply partial differential equation to the expression (8) as follows:

$$
\frac{\partial^{2} \varphi}{\partial x^{2}}=\frac{1}{C_{o}^{2} T} \frac{\partial^{2} \varphi}{\partial t^{2}} .
$$

From equation (9) we obtain

$$
\frac{1}{\varphi} \frac{d^{2} \varphi}{d x^{2}}=\frac{1}{C_{o}^{2} T} \frac{d^{2} T}{d t^{2}} .
$$

Now, if we let $\frac{1}{\varphi} \frac{d^{2} \varphi}{d x^{2}}+p^{2}=0$ which can be re-written as:

$$
\frac{d^{2} \varphi}{d x^{2}}+\varphi \varphi^{2}=0
$$

Then we will observe that the solution of equation (11) is of the form

$$
\phi=A \sin (p x)+\mathrm{B} \cos (p x)(A \text { and } B \text { are constants }) .
$$

Similarly, if we let $\frac{1}{C_{o}^{2} T} \frac{d^{2} \varphi}{d t^{2}}+q^{2}=0$, we obtain similar solution to (12) and get equation:

$$
T=E \sin \left(q C_{0} t\right)+D \cos \left(q C_{0} t\right),
$$

where $E$ and $D$ are constants.

Now we observe that equation (12) and (13) have functions of $x$ and $t$ respectively. If we combine the two functions, we can have a new expression:

$$
\begin{array}{r}
\phi(x, t)=(A \sin (p x)+B \cos (p x)) \times \\
\left(E \sin \left(q C_{0} t\right)+D \cos \left(q C_{0} t\right)\right) .
\end{array}
$$

By setting the boundary conditions at zero so that we have $x=0, j=0$, equation (14) is reduced to the form:

$$
0=B\left(E \sin \left(q C_{0} t\right)+D \cos \left(q C_{0} t\right)\right) .
$$

Now, if equation (14) is revisited and $B=0$ is substituted in the expression, then equation (14) becomes:

$$
\phi(x, t)=A \sin (p x)\left(E \sin \left(q C_{0} t\right)+D \cos \left(q C_{0} t\right)\right) .
$$

When the term " $\mathrm{A}$ " is brought to inside the bracket, equation (16) changes its form to become:

$$
\phi(x, t)=\sin (p x)\left(A E \sin \left(q C_{0} t\right)+A D \cos \left(q C_{0} t\right)\right) .
$$

If we represent $A E$ by $Q_{r}$, and $A D$ by $P_{r}$, then equation (17) becomes

$$
\phi(x, t)=\sin (p x)\left(Q_{r} \sin \left(q C_{0} t\right)+P_{r} \cos \left(q C_{0} t\right)\right) .
$$

If we consider point $x=d$ (where $d>C$ ) and $\varphi$ $=0$, then we have

$$
0=\sin (p d)\left(Q_{r} \sin (q C t)+P_{r} \cos (C t)\right) .
$$

If $\sin (p d)$ is now equaled to zero, $P d=\sin ^{-1}(0)$

This gives $\quad P d=n p($ where $n=1,2 \ldots)$.

Thus, $P=\frac{n \pi}{d}$.

Now, if we let $q=P$, and $c q=\alpha$, while noting that $C_{0}$ is a constant for any wave, then we have the following equation:

$$
\phi(x, t)=\sin \frac{n \pi}{d} \mathrm{x}\left(\mathrm{Q}_{\mathrm{r}} \sin \left(p C_{0} t\right)+P_{r}\left(p C_{0} t\right)\right) .
$$

Now,

$$
\begin{aligned}
\phi(x, t)= & \sum_{r=1}^{\infty} \phi(x, t)= \\
& \sum_{r=1}^{\infty} \sin \frac{r \pi}{d} \mathrm{x}\left(\mathrm{Q}_{\mathrm{r}} \sin \left(p C_{0} t\right)+P_{r}\left(p C_{0} t\right)\right) .
\end{aligned}
$$

If we set $t=0$,

$$
g(x)=\sum_{r=1}^{\infty} P_{r} \sin \frac{r \pi}{d} x .
$$

However, we know that from Fourier series $P_{r}=$ $2 x$ (mean value) of $g(x) \sin \frac{r \pi}{d} x$.

If $\mathrm{x}$ ranges from 0 to $\mathrm{e}$, then we have

$$
P_{r}=\frac{2}{C} \int_{0}^{c} h(x) \sin \frac{r \pi}{d} x d x .
$$

By partial differentiation

$$
\left(\frac{\partial \varphi}{\partial t}\right)_{t=0} h(x)=\sum_{r=1}^{\infty} Q r \sin \left(\frac{r \pi}{\varphi}\right) x P c
$$

but $P C_{0} Q_{r}=2 \mathrm{x}$ (mean value) of $h(x) \sin \left(\frac{r \pi}{d}\right) x$

If we let $\mathrm{x}$ range from 0 to $\mathrm{C}$,

then we have $P C_{0} Q_{r}=\frac{2}{C} \int_{0}^{c} h(x) \sin \left(\frac{r \pi}{d}\right) x d x$.

But $P=\frac{n \pi}{d}$, therefore

$$
Q_{r}=\frac{2 d}{n \pi \pi C_{o}} \int_{0}^{c} h(x) \sin \left(\frac{r \pi}{d}\right) x d x .
$$



tion:

We can then express $j(x, t)$ as follows in equa-

$$
\begin{aligned}
& \varphi(x, t)=\sum_{e=1}^{\infty} \varphi(x, t) \sum_{r=1}^{\infty} \sin \frac{n \pi}{d} x \times \\
& {\left[\left(\frac{2 d}{n \pi c C_{0}} \int_{0}^{c} h(x) \sin \left(\frac{r \pi}{d}\right) x d x\right) \sin \frac{n \pi}{d} C_{0} t\right)+} \\
& \left(\frac{2}{c} \int_{0}^{c} g(x) \sin \left(\frac{r \pi}{d}\right) x d x \cos \frac{r \pi}{d}\right)
\end{aligned}
$$

Finally, the expression relating velocity is then expressed as follows:

$$
\begin{aligned}
& V(x, t)=f_{0} \lambda_{0} \sum_{r=1}^{\infty} \frac{n \pi}{d} x \times \\
& {\left[\left(\frac{2 d}{n \pi c C_{0}} \int_{0}^{c} h(x) \sin \left(\frac{r \pi}{d}\right) x d x\right) \times\right.} \\
& \left.\sin \left(\frac{n \pi}{d} c_{0}\right) t+\left(\frac{2}{c} \int_{0}^{c} g(x) \sin \left(\frac{r \pi}{d}\right) x d x \cos \frac{r \pi}{d}\right)^{t}\right],
\end{aligned}
$$

Table 1. Randomly generated values of parameters

\begin{tabular}{ccccccccc}
\hline Iteration & $Q$ & $P r$ & $t$ & $d$ & $f_{0}$ & $L$ & $x$ & $e$ \\
\hline 1 & 8.380854 & 3.545605 & .6172907 & 2.299296 & 41.25132 & .3235891 & .6966043 & 2.188447 \\
2 & 6.435208 & 9.004733 & $3.79 \times 10^{-02}$ & 1.239815 & 60.81092 & 1.529529 & .2115946 & .6647442 \\
3 & 7.128839 & 8.217785 & .1588004 & 2.97932 & 25.19123 & .6286248 & $3.94 \times 10^{-02}$ & .1237937 \\
4 & 3.506084 & 6.855216 & .8544856 & 1.181925 & 37.56111 & .2469058 & .288271 & .9056299 \\
5 & 9.737608 & 9.104248 & .952101 & 4.517972 & 41.00356 & .3267362 & .7094617 & 2.22884 \\
6 & 6.340928 & 4.307105 & .8452433 & .7569605 & 63.35018 & .6935805 & .170283 & .5349597 \\
7 & .5259353 & 8.459236 & .3425378 & 3.530533 & 96.98044 & 1.831649 & .2818047 & .8853157 \\
\hline
\end{tabular}

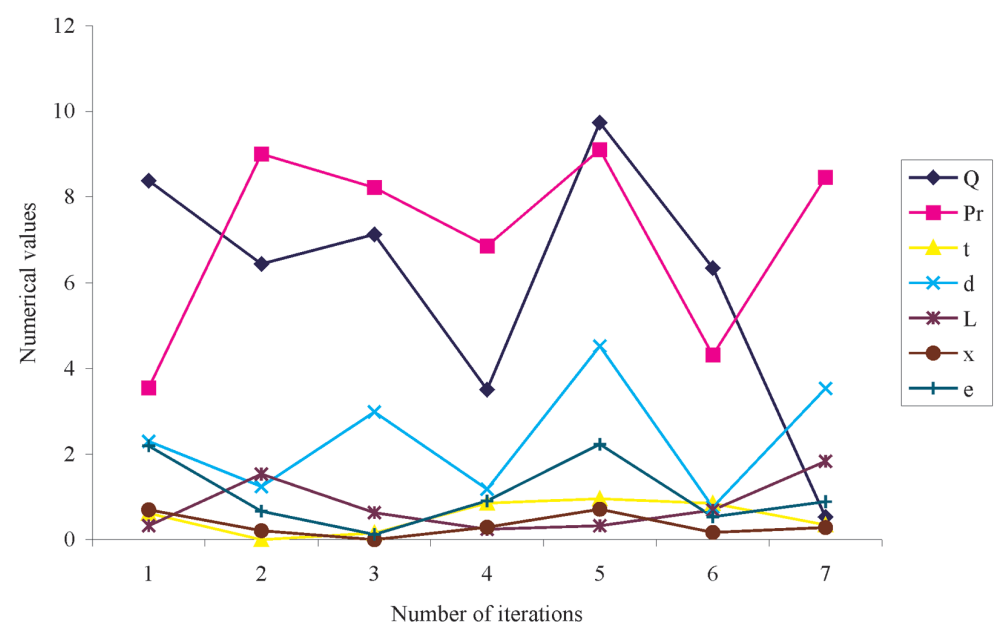

Fig 2. The data for $Q, P_{r}, t, d L, x$ and $e$ 


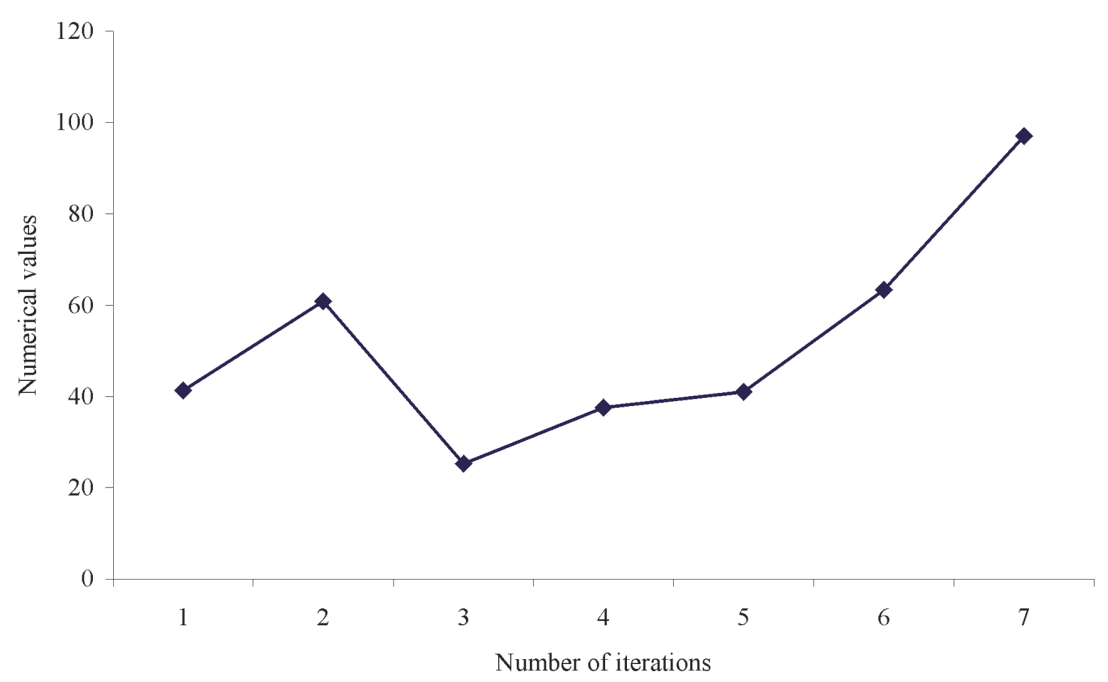

Fig 3. The data for $f_{0}$

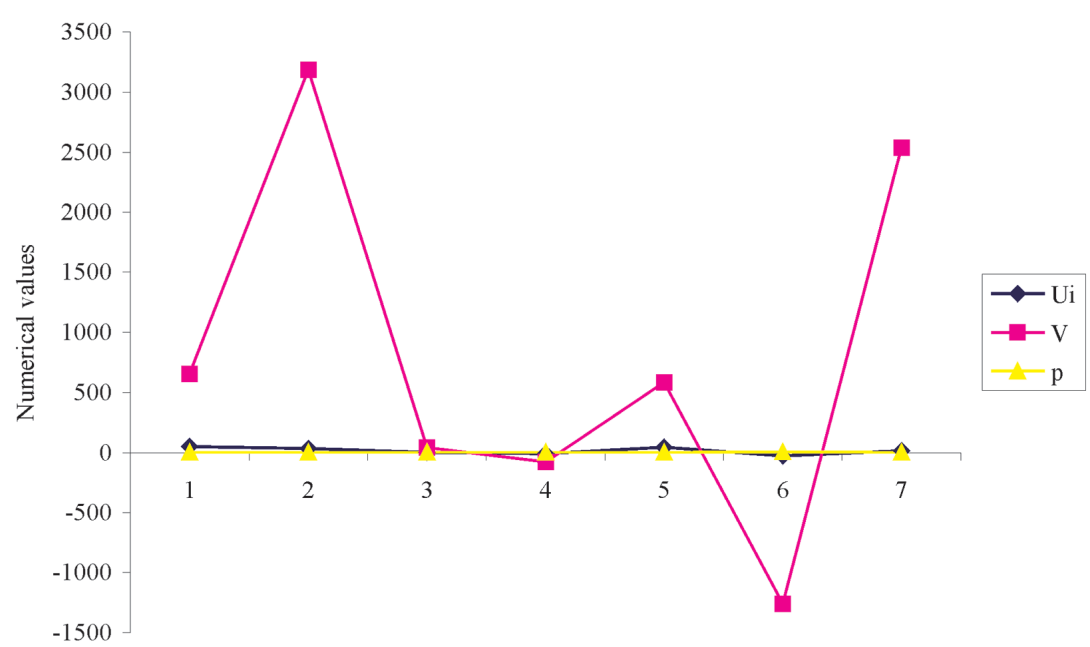

Number of iterations

Fig 4. $U_{i}, V$ and $p$

Table 2. Output values for each iteration

\begin{tabular}{|c|l|l|l|}
\hline Iteration & \multicolumn{1}{|c|}{$\mathrm{U}_{\mathrm{i}}$} & \multicolumn{1}{c|}{$\mathrm{V}$} & \multicolumn{1}{c|}{$\mathrm{p}$} \\
\hline 1 & 49.12919 & 655.7999 & 1.366328 \\
2 & 34.26075 & 3186.665 & 2.53392 \\
3 & 2.701572 & 42.78165 & 1.054466 \\
4 & -8.436416 & -78.23979 & 2.65803 \\
5 & 43.64706 & 584.7548 & .6953547 \\
6 & -28.60369 & -1256.802 & 4.150273 \\
7 & 14.29041 & 2538.464 & .8898352 \\
\hline
\end{tabular}

\section{References}

1. Berry, M. J.; Koves, T. R. and Benedetto, J. J. The influence of speed, grade and mass during simulated off road bicycling. Applied Egronomics, Vol 31, Issue 5, 2000. p. 445-546.

2. Das, K. S.; Srivastava, B. B. and Das, N. Standing orientation and behaviour of goats during short-haul road transportation. Small Ruminant Research, Vol 41, Issue 1, 2001, p. 91-94.

3. Dieffenbachia, J.; Lynch, R. S.; Octting, E. R. and Swain, R. C. The Driving Anger Expression Library: a measure of how people express their anger on the road. Behaviour and Research Therapy, Vol 40, Issue 6, 2002, p. 717-737.

4. Kim, H.; Yang, H. S. and Park, Y. P. Improving the vehicle performance with active suspension using road serving algorithm. Computers and Structures, Vol 80, Issue 18-19, 2002, p. 1569-1577.

5. Kojima, Y.; Maeda, K.; Ohta, H. and Maruta, O. Effects of aerodynamics on a high-speed vehicle: 
aeroclastic analysis of longitudinal motion". ISAE Review, Vol 16, Issue 3, 1995, p. 292-293.

6. Lee, H. C.; Cameron, D. and Lee, A. H. Assesing the driving performance of older adult divers: on-road versus simulated driving. Accident Analysis and Prevention, Vol 35, Issue 5, 2003, p. 631-823.

7. Nande, A. F, and Sugman, J. A. Optimization of road vehicle passive suspension systems. Part 1. Optimization algorithm and vehicle model. Applied Mathematical Modeling, Vol 27 Issue 4, 2003, p. 249-261.
8. Salau, T. A. O.; Adeyefa, O. A.; Oke, S. A. Vehicle speed control using road bumps. Transport, Vol XIX, No 3, 2004, p. 130-136, ISBN 1648-4142, http:/www.vtu.lt/ english/editions

9. Rubinstein, D and Hitron, R. A detailed multi-body model for dynamic simulation of off-road tracked vehicles. Journal of Terramechanics, Vol 41, Issue 2-3, 2004, p. $69-185$. 